\title{
Risk factors of atherosclerosis during systemic therapy targeting vascular endothelial growth factor
}

\author{
HANA ŠTUDENTOVÁ ${ }^{1}$, JARMILA INDRÁKOVÁ ${ }^{2}$, PAVLA PETROVÁ ${ }^{3}$, MILAN KAMÍNEK ${ }^{4}$, \\ HANA KALÁBOVÁ ${ }^{1}$, VLASTISLAV ŠRÁMEK ${ }^{1}$, TOMÁŠ ADAM ${ }^{3}$ and BOHUSLAV MELICHAR ${ }^{1,5}$ \\ Departments of ${ }^{1}$ Oncology, ${ }^{2}$ Medicine, ${ }^{3}$ Clinical Biochemistry and ${ }^{4}$ Nuclear Medicine; \\ ${ }^{5}$ Institute of Molecular and Translational Medicine, Palacký University Medical School and Teaching Hospital, \\ Olomouc 775 20, Czech Republic
}

Received November 10, 2014; Accepted September 9, 2015

DOI: $10.3892 / 01.2015 .4017$

\begin{abstract}
The aim of the present study was to examine the changes in intima-media thickness (IMT) and myocardial perfusion in association with other laboratory risk factors for atherosclerosis in patients treated with therapy that targeted vascular endothelial growth factor (VEGF). IMT, myocardial perfusion and laboratory risk factors of atherosclerosis were studied in 58 patients with metastatic colorectal carcinoma or metastatic renal cell carcinoma prior to and at 3-monthly intervals during anti-VEGF treatment. Compared with the pretreatment IMT, the results indicated that the IMT was consistently increased during therapy in the two patient groups. Patient blood pressure and concentration of troponin $\mathrm{T}$ increased transiently. An increase in the concentration of high-density lipoprotein cholesterol and decrease in the concentrations of C-reactive protein and homocysteine were also observed. Novel myocardial ischemia was evident in individual patients. In conclusion, anti-VEGF therapy affects the laboratory risk factors of atherosclerosis and results in an acceleration of atherosclerosis, as demonstrated by increased IMT.
\end{abstract}

\section{Introduction}

Targeted therapy has almost transformed the management of patients with advanced cancer. Targeted therapy aims to affect one or more of the hallmarks of cancer, which are the pathogenic mechanisms responsible for tumor growth and progression (1). Angiogenesis is indispensable for tumor progression and metastasis, and vascular endothelial growth factor (VEGF) is the key mediator of angiogenesis (2).

Correspondence to: Professor Bohuslav Melichar, Department of Oncology, Palacký University Medical School and Teaching Hospital, 6 I.P. Pavlova, Olomouc 775 20, Czech Republic

E-mail: bohuslav.melichar@fnol.cz

Key words: atherosclerosis, biomarkers, intima-media thickness, single-photon emission computed tomography
Anti-VEGF agents, which currently include the monoclonal antibodies bevacizumab and ramucirumab, recombinant protein aflibercept or low-molecular weight inhibitors sunitinib, axitinib, sorafenib and regorafenib, are used across a wide spectrum of various advanced tumors (3-10). Targeted therapy exhibits selective action on tumor cells or other tissues that are essential for tumor progression. Targeted therapy is considered to exert minimal effects on normal tissues. Unfortunately, targeted therapy is not free of side-effects. A novel spectrum of side-effects has emerged with the advent of targeted agents, including skin toxicity, hypomagnesemia and disorders of glucose and lipid metabolism $(11,12)$.

Consideration of the toxicity of systemic treatment in patients with advanced cancer has previously been limited to acute side-effects, such as myelosuppression or gastrointestinal toxicity (13-15). With the improved survival and, in extreme cases, successful treatment of patients with advanced solid tumors, chronic toxicity has emerged as an extremely important issue (16). One of the most important long-term consequences of systemic therapy is the increased risk of atherosclerosis and the associated complications. An increased rate of atherosclerosis has been described in patients with germinal tumors (17-19), and similar findings have also been reported in patients with breast cancer (20).

The most prominent side-effects of anti-VEGF therapy, such as hypertension and proteinuria, are also associated with an increased risk of the atherosclerosis progression. However, the clinical data on the impact of anti-VEGF agents on the progression of atherosclerosis remain limited (21).

The aim of the present study was to examine the changes in intima-media thickness (IMT), a surrogate biomarker for the presence of atherosclerosis, in association with other laboratory risk factors of atherosclerosis in cancer patients treated with anti-VEGF therapy.

\section{Patients and methods}

Patients. In total, 58 patients with metastatic colorectal carcinoma (mCRC) or metastatic renal cell carcinoma (mRCC) that were consecutively scheduled to be treated with agents targeting the VEGF pathway were enrolled in the present pilot study between July 2008 and December 2009 at Palacký University 
Medical School and Teaching Hospital (Olomouc, Czech Republic). Out of the patients with $\mathrm{mCRC}, 44$ patients were treated with a combination of bevacizumab $(5 \mathrm{mg} / \mathrm{kg} / 30 \mathrm{~min})$ and folinic acid (50 mg bolus), fluorouracil (400 mg/m $\mathrm{m}^{2}$ bolus followed by $2400 \mathrm{mg} / \mathrm{m}^{2}$ given as a continuous infusion over $46 \mathrm{~h}$ ) and oxaliplatin (FOLFOX)7 (130 mg/m²/2 h), 2 patients were treated with bevacizumab $(5 \mathrm{mg} / \mathrm{kg} / 30 \mathrm{~min})$ plus folinic acid ( $50 \mathrm{mg}$ bolus), fluorouracil $\left(400 \mathrm{mg} / \mathrm{m}^{2}\right.$ bolus followed by $2400 \mathrm{mg} / \mathrm{m}^{2}$ given as a continuous infusion over $46 \mathrm{~h}$ ) and irinotecan $\left(180 \mathrm{mg} / \mathrm{m}^{2} / 90 \mathrm{~min}\right)$ and 1 patient was treated with bevacizumab $(5 \mathrm{mg} / \mathrm{kg} / 30 \mathrm{~min}$ ) plus FOLFOX6 (folinic acid, $50 \mathrm{mg}$ bolus; fluorouracil, $400 \mathrm{mg} / \mathrm{m}^{2}$ bolus followed by $2400 \mathrm{mg} / \mathrm{m}^{2}$ given as continuous infusion over $46 \mathrm{~h}$ and oxaliplatin $100 \mathrm{mg} / \mathrm{m}^{2} / 2 \mathrm{~h}$ ). All 11 patients with $\mathrm{mRCC}$ were treated with sunitinib given as $50 \mathrm{mg}$ once daily on a 4 - 2 schedule (4 weeks of treatment followed by 2 -week intervals without therapy). The protocol of the investigations was approved by the Institutional Ethics Committee at the Palacký University Medical School and Teaching Hospital, and the patients provided informed consent.

Laboratory examinations. Blood samples were drawn from a peripheral vein subsequent to an overnight fast. The samples were transferred immediately to the laboratory and centrifuged at $1,600 \times \mathrm{g}$ for $8 \mathrm{~min}$ at $14^{\circ} \mathrm{C}$. The serum and plasma were separated and analyzed immediately or frozen at $-20^{\circ} \mathrm{C}$ until analysis. Glucose, uric acid, C-reactive protein (CRP), total cholesterol, triglycerides, lipoprotein(a) and high-density lipoprotein (HDL) cholesterol were determined using commercially available kits on the Cobas 8000 system (Roche Diagnostics, Mannheim, Germany), according the manufacturer's instructions. Low-density lipoprotein (LDL) cholesterol was calculated using the following formula: LDL cholesterol $=$ total cholesterol - triglycerides/2.2 $-\mathrm{HDL}$ cholesterol. Glycosylated hemoglobin was determined by high performance ion-exchange liquid chromatography using Arkray Adams HA-8180V analyzer (Arkray, Kyoto, Kyoto, Japan) (22). Determination of the total homocysteine levels in the plasma was performed by high performance liquid chromatography (HPLC) with fluorescence detection performed at $385 \mathrm{~nm}$ excitation and $515 \mathrm{~nm}$ emission using the HPLC HP 1100 system (Hewlett-Packard, Waldbronn, Germany), as previously reported (22). The measurement of creatinine levels was performed using an enzymatic method with photometric detection, using a commercially available kit on Cobas 8000 system. Myoglobin, N-terminal pro-brain natriuretic peptide (NT-proBNP) and troponin T concentrations were determined by electro-chemiluminiscence immunoassay, using commercially available kits on the Cobas 8000 system. To measure troponin $\mathrm{T}$, the high sensitivity assay for cardiac troponin $\mathrm{T}$ was used.

To assess IMT, carotid arteries were evaluated by two-dimensional imaging using the Philips iE33 ultrasound scanner (Philips Medical Systems, Bothell, WA, USA) with a 3-10 MHz transducer, as previously described (22). The IMT, which was defined as the distance between the echogenic lines representing blood-intima interface and media-adventitia interface, was measured on the posterior wall of the carotid artery in the longitudinal plane and evaluated bilaterally at two levels. Three measurements were performed at each level by the same observer, and the mean of these measurements was calculated.

Ambulatory blood pressure monitoring was obtained using a fully automatic ambulatory monitor (ABPM-04; Meditech, Budapest, Hungary) with an oscillometric technique that recorded blood pressure and recorded readings every $30 \mathrm{~min}$ throughout the day and night for $24 \mathrm{~h}$, while patients maintained a normal routine.

Imaging examinations. Myocardial perfusion was assessed using single-photon emission computed tomography (SPECT) with ${ }^{99 m}$ technetium sestamibi. SPECT was performed using a Siemens e.CAM camera (Siemens Medical Solutions, Erlangen, Germany) equipped with a $90^{\circ}$ angled dual head and low-energy high-resolution collimators. The images were gated at 8 frames/cardiac cycle. No attenuation correction was applied. Additional prone position imaging was used in the case of inferior wall defect, which was considered to be caused by attenuation artifact. The summed stress score (SSS) and summed difference score (SDS) of myocardial perfusion, left ventricular ejection fraction (EF) and end-diastolic/end-systolic volumes (EDV/ESV) were calculated automatically using the 4D-MSPECT software (University of Michigan, Ann Arbor, MI, USA).

Patients underwent 1-day imaging at rest with nitrate administration, during which $8 \mathrm{mCi}$ (296 MBq) ${ }^{99 \mathrm{~m}}$ technetium sestamibi (MIBI scan) was injected at rest, with SPECT imaging performed $1 \mathrm{~h}$ subsequent to injection. Stress testing was performed on a separate day using ${ }^{99} \mathrm{~m}$ technetium sestamibi. Patients either underwent an exercise test or received intravenous dipyridamole if unable to exercise. The exercise test was performed upright on a bicycle ergometer. Each patient exercised until $85 \%$ of the age-predicted maximal heart rate was reached, or until the onset of angina pectoris, dyspnea or fatigue, dizziness, frequent multifocal or paired ventricular extrasystoles ( $>10 / \mathrm{min})$, ST segment depression $(>0.2 \mathrm{mV})$, or a decrease in blood pressure of $10 \mathrm{mmHg}$ below the peak value of the previous stage. Dipyridamole was administered intravenously at a standard dose of $0.56 \mathrm{mg} / \mathrm{kg}$ of body weight during a 4-min period in the combination with low level of exercise in patients that could not achieve the maximal predicted heart rate. Dipyridamole stress alone was used for patients with a left bundle branch block.

Statistical analysis. Differences between subgroups of patients were analyzed by the Mann-Whitney U test. Correlations were analyzed using Spearman's rank correlation coefficient (rs). Differences in the parameters during the course of therapy were analyzed using the Wilcoxon signed-rank test. $\mathrm{P}<0.05$ was considered to indicate a statistically significant difference. The analyses were performed using NCSS software (Number Cruncher Statistical Systems, Kaysville, UT, USA). The data were expressed as the mean \pm standard deviation, where appropriate.

\section{Results}

Patient characteristics. A total of 58 patients, consisting of 21 females and 37 males aged $62 \pm 8$ years (range, 37-76 years) that were diagnosed with either $\operatorname{mCRC}(n=47)$ or $\mathrm{mRCC}$ 
$(n=11)$, were assessed in the present study. Compared with the patients with $\mathrm{mCRC}$, the patients with $\mathrm{mRCC}$ demonstrated a slightly increased troponin T level (mRCC, $0.012 \pm 0.004 \mu \mathrm{g} / \mathrm{l}$; $\mathrm{mCRC}, 0.011 \pm 0.0002 \mu \mathrm{g} / \mathrm{l} ; \mathrm{P}=0.026)$, myoglobin concentration (mRCC, 53 $\pm 31 \mu \mathrm{g} / \mathrm{l} ; \mathrm{mCRC}, 35 \pm 14 \mu \mathrm{g} / \mathrm{l} ; \mathrm{P}=0.048)$ and creatinine $(\mathrm{mRCC}, 93 \pm 18 \mu \mathrm{mol} / \mathrm{l} ; \mathrm{mCRC}, 78 \pm 18 \mu \mathrm{mol} / \mathrm{l}$; $\mathrm{P}=0.027)$ concentrations and significantly lower total cholesterol level (mRCC, 4.27 $\pm 0.96 \mathrm{mmol} / 1$; mCRC, $5.15 \pm 1.23 \mathrm{mmol} / \mathrm{l} ; \mathrm{P}=0.043)$ and LDL cholesterol level (mRCC, $2.50 \pm 0.82 \mathrm{mmol} / 1 ; \mathrm{mCRC}, 3.41 \pm 1.09 \mathrm{mmol} / \mathrm{l} ; \mathrm{P}=0.017$ ), but no other significant differences were noted between the patients with $\mathrm{mCRC}$ and mRCC. A decreasing number of measurements were obtained during the course of therapy. The principal reason for the decreasing number of measurements was disease progression or declining condition of the patient, which was also associated with a diminished compliance with the follow-up protocol. Therefore, the measurements of IMT and metabolic parameters could be obtained in $<50 \%$ of patients on subsequent visits, and the number of subjects examined at visit 4 was considerably smaller.

Changes in parameters of atherosclerosis. Compared with the pretreatment baseline, IMT was consistently significantly increased at subsequent visits (Table I). Systolic and diastolic blood pressure were each increased transiently at visit 2 , but returned to normal on subsequent visits. A transient significant increase at visit 2 was also observed for troponin T levels. The total cholesterol level was increased at visits 2 and 3, and this increase was due to a significant increase in the concentration of HDL cholesterol. A transient statistically significant decrease in the concentrations of CRP $(\mathrm{P}=0.023)$ and homocysteine $(\mathrm{P}=0.0004)$ was observed at visit 2 . The decrease in serum creatinine concentrations was of borderline statistical significance.

SPECT at baseline was performed in 55 patients, and ischemia was observed in 6 of these patients. Control SPECT was performed subsequent to 3 and 6 months in 24 and 8 patients, respectively. Novel symptoms of ischemia were observed in 2 patients subsequent to 3 months and in additional 3 patients subsequent to 6 months. In addition, 1 patient with $\mathrm{mCRC}$ that demonstrated originally normal SPECT findings suddenly succumbed during the administration of the third cycle of bevacizumab plus FOLFOX7. The autopsy identified acute myocardial infarction as the cause of mortality.

The significant increase of IMT was also evident when mCRC (visit 2, $0.74 \pm 0.09 \mathrm{~mm}$; baseline, $0.70 \pm 0.10 \mathrm{~mm}$; $\mathrm{P}=0.015$ ) and $\mathrm{mRCC}$ (visit 2, 0.83 $\pm 0.11 \mathrm{~mm}$; baseline, $0.71 \pm 0.11 \mathrm{~mm} ; \mathrm{P}=0.028$ ) patients were evaluated separately at visit 2. This increase was evident on subsequent visits as well. In patients with mCRC, the changes in blood pressure at visit 2, HDL cholesterol at visits 2 and 3, lipoprotein(a) and CRP at visit 2, homocysteine at visits 2 and 3 also reached statistical significance when evaluated separately. The decrease in the serum creatinine level at visit 2 was highly significant in $\mathrm{mCRC}$ patients (visit 2, 73 $\pm 17 \mu \mathrm{mol} / \mathrm{l}$; baseline, $78 \pm 18 \mu \mathrm{mol} / 1 ; \mathrm{P}=0.002$ ). In addition, at visit 2 , a transient significant increase of myoglobin (visit 2, 109 $\pm 321 \mu \mathrm{g} / \mathrm{l}$; baseline, $39 \pm 18 \mu \mathrm{g} / \mathrm{l} ; \mathrm{P}=0.034)$ and a significant decrease of uric acid (visit 2, 286 $\pm 59 \mu \mathrm{mol} / 1$; baseline, 306 $\pm 73 \mu \mathrm{mol} / 1$; $\mathrm{P}=0.046$ ) was evident in mCRC patients. In mRCC patients, an increase of HDL concentrations at visit 3 was also evident.
Correlation between IMT and parameters of atherosclerosis. At baseline, IMT demonstrated a significant positive correlation with myoglobin ( $\mathrm{rs}=0.416 ; \mathrm{P}=0.005)$ and a negative correlation with lipoprotein (a) (rs=-0.392; $\mathrm{P}=0.010)$. At visit 3 , subsequent to 6 months, IMT demonstrated a significant negative correlation with serum HDL cholesterol ( $\mathrm{rs}=-0.566$; $\mathrm{P}=0.044)$. No other significant correlations between IMT and the laboratory parameters of atherosclerosis risk were observed on subsequent visits. CRP at baseline exhibited a significant negative correlation with diastolic blood pressure ( $\mathrm{rs}=-0.282 ; \mathrm{P}=0.041)$, HDL cholesterol ( $\mathrm{rs}=-0.341 ; \mathrm{P}=0.015)$ and creatinine ( $\mathrm{rs}=-0.289 ; \mathrm{P}=0.037)$, and a positive correlation with NT-proBNP ( $\mathrm{rs}=0.468 ; \mathrm{P}=0.0007$ ). At visit 2, subsequent to 3 months, CRP was negatively correlated with HDL cholesterol ( $\mathrm{rs}=-0.402 ; \mathrm{P}=0.051)$ and positively correlated with NT-proBNP ( $\mathrm{rs}=0.506 ; \mathrm{P}=0.012$ ). At visit 3 , a significant negative correlation between diastolic blood pressure and CRP was identified ( $\mathrm{rs}=-0.574 ; \mathrm{P}=0.010$ ). The limited number of subjects examined prevented the assessment of correlation at visit 4 , which occurred subsequent to 12 months.

\section{Discussion}

The present data demonstrate that the administration of anti-VEGF therapy is associated with a marked and rapid increase in IMT, which acts as a surrogate biomarker of atherosclerosis. In addition, myocardial ischemia manifested on control SPECT or clinically in certain patients. The increase in the concentration of troponin $\mathrm{T}$ and myoglobin indicates an even higher prevalence of myocardial damage during the first 3 months of therapy. By contrast, the changes in the laboratory biomarkers of atherosclerosis risk during the treatment were more complex with certain changes, such as increased systolic and diastolic blood pressure or lipoprotein(a) concentration, indicating an increased risk of atherosclerosis, while other laboratory biomarkers, such as HDL cholesterol, homocysteine or CRP, indicated a decreased risk. However, no significant change was observed in the majority of laboratory biomarkers of atherosclerosis risk that were assessed in the present study.

The significant increase in systolic and diastolic blood pressure was evident only at the visit subsequent to 3 months of therapy, as was the increase in the concentrations of troponin $\mathrm{T}$ and myoglobin, which indicated myocardial injury. The absence of an increase in blood pressure on subsequent visits may also be due to either the anti-VEGF therapy being discontinued or antihypertensive medication being adjusted. Similar to the earlier study performed in mCRC patients treated with bevacizumab combined with the FOLFOX7 regimen, a decrease in CRP and homocysteine was observed in the present study (21). In contrast to the previous study in patients treated with bevacizumab plus FOLFOX7, no increase of glycosylated hemoglobin was evident in the current study. Circulating concentrations of CRP and homocysteine may be associated with the tumor mass and progression, and CRP and homocysteine acted as tumor biomarkers in the present study, as demonstrated by a significant decrease in CRP and homocysteine subsequent to 3 months of treatment. Also, in accordance with previous studies (21), an increase of HDL cholesterol was observed during anti-VEGF therapy. A negative correlation has been observed between CRP and 


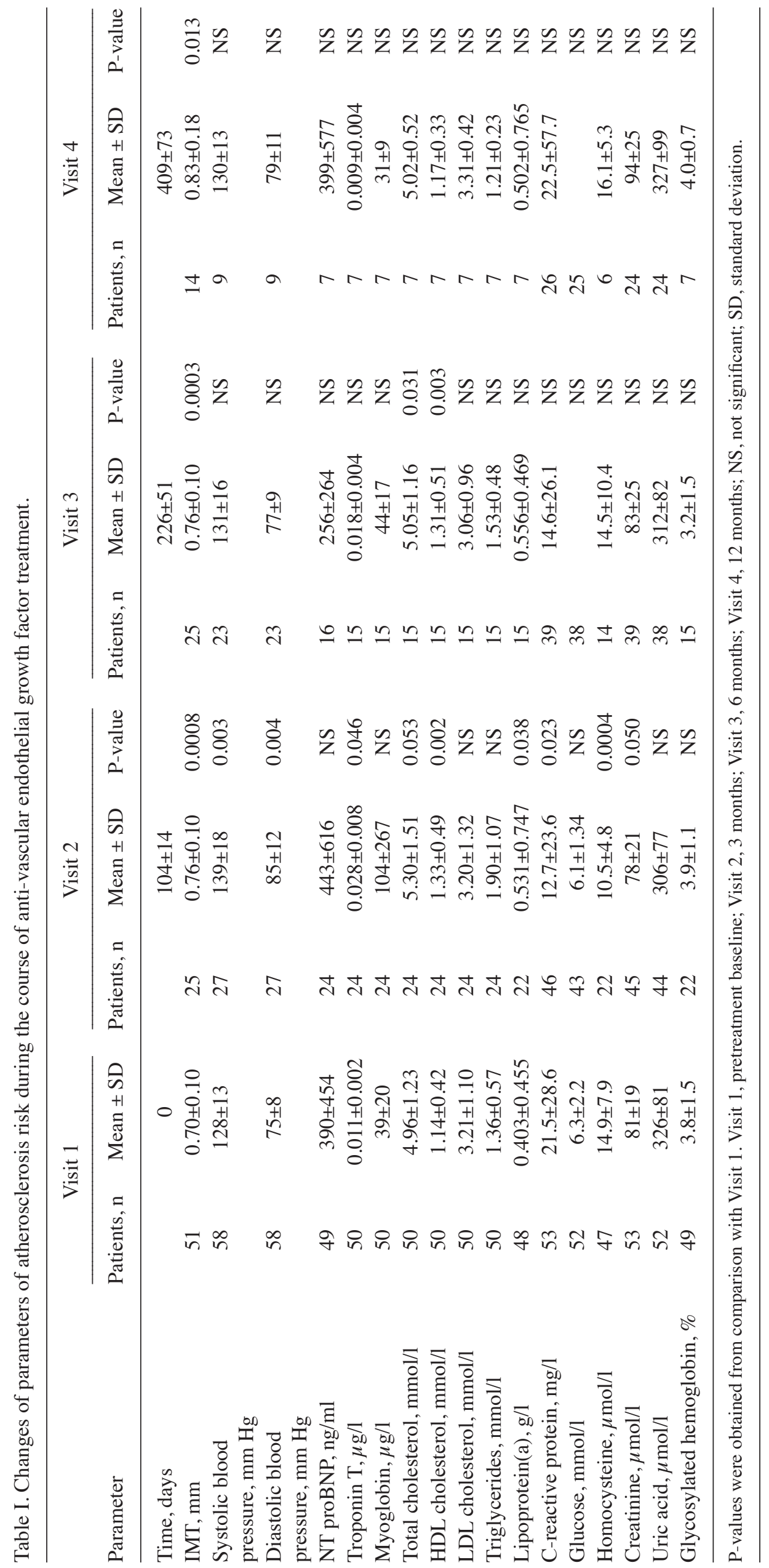


HDL cholesterol. An increase in HDL cholesterol during the treatment may be associated with the effect of therapy on serum CRP levels. From this perspective, the negative correlation between HDL cholesterol and IMT observed during the course of interest is of specific interest.

Laboratory assessment plays an important role in the management of cancer patients (23). The present study demonstrates the potential utility of laboratory biomarkers for atherosclerosis in patients treated with targeted therapy. In general, the biomarkers for atherosclerosis behaved similarly in $\mathrm{mCRC}$ and mRCC patients. Subsequent to splitting the cohort according to diagnosis, the number of subjects evaluated was even more limited, and the results of these analyses, particularly when the differences were not significant, should be regarded with caution. The marked increase of myoglobin concentrations may also be associated with the toxicity of the chemotherapy regimen administered concomitantly with bevacizumab.

Cardiac toxicity has been known to accompany the administration of cytotoxic drugs for numerous decades (24). Originally, cardiotoxicity was hypothesized to encompass only a direct toxic effect of the anticancer drugs on the myocardium. Only more recently was it hypothesized that cytotoxic chemotherapy may also induce cardiotoxicity indirectly by accelerating the progression of atherosclerosis (17-20). Advanced cancer and atherosclerosis, with the associated complications, are each associated with systemic immune and inflammatory responses (25-27), and the systemic inflammatory response induced by the tumor or therapy may result in accelerated atherosclerosis. It has been demonstrated that IMT increases subsequent to cytotoxic chemotherapy in patients with breast cancer (20). Among targeted agents, cardiac toxicity represents a well-characterized side-effect of trastuzumab (28). More recently, cardiac toxicity has also been described for anti-VEGF agents in MRCC (29). In the present study, an increase of IMT, an indicator of atherosclerosis, was evident in mCRC patients treated with the combination of chemotherapy and bevacizumab and also in mRCC patients treated with sunitinib, indicating that accelerated atherosclerosis may represent a class effect of anti-VEGF therapy. Despite the limited size of the cohort, a statistically significant IMT increase was apparent even when mRCC patients were analyzed separately. Compared to the anthracycline cardiotoxicity that is rare below a certain cumulative dose, or trastuzumab cardiotoxicity that is reversible, the rapid IMT increase during anti-VEGF therapy may represent a specific challenge. The insidious nature of this toxicity may be illustrated by the case of a patient that succumbed to acute myocardial infarction during the present study. The advent of targeted therapy resulted in marked prolongation of overall survival, notably in patients with $\mathrm{mCRC}$ or $\mathrm{mRCC}$. In addition, multiple lines of anti-VEGF therapy are available in each indication (3-7). With additional improvement in prognosis, the identification of biomarkers for atherosclerosis may become an important issue in this patient population.

The present study has evident limitations. First, the cohort of patients investigated included patients with two different tumors treated with different agents, and was also extremely heterogeneous with regard to the age of patients. The patients with $\mathrm{mCRC}$, which comprised the majority of patients, were treated by the combination of bevacizumab with chemotherapy, and the effects of the anti-VEGF agent and chemotherapy could not be isolated in the present study. Repeat measurements were performed only in a proportion of patients. By contrast, from the perspective of long-term complications, responding patients are the most notable subgroup. The survival of patients with advanced tumors, such as $\mathrm{mCRC}$ or $\mathrm{mRCC}$, has increased markedly over the last decade, and the issue of chronic toxicity can no longer be neglected (30).

In conclusion, the present data indicate that anti-VEGF therapy affects the laboratory risk factors of atherosclerosis and also results in an acceleration of atherosclerosis. Future studies should address potential preventive measures in patients with expected long-term survival.

\section{Acknowledgements}

The present study was supported by grant from the Ministry of Education, Youth and Sports of the Czech Republic (no. LO1304) and from Palacký University (no. RVO61989592).

\section{References}

1. Hanahan D and Weinberg RA: Hallmarks of cancer: The next generation. Cell 144: 646-674, 2011.

2. Hanahan D and Weinberg RA: The hallmarks of cancer. Cell 100: 57-70, 2000.

3. Buchler T, Klapka R, Melichar B, Brabec P, Dusek L, Vyzula R and Abrahamova J: Sunitinib followed by sorafenib or vice versa for metastatic renal cell carcinoma - data from the Czech registry. Ann Oncol 23: 395-401, 2012.

4. Rini BI, Escudier B, Tomczak P, Kaprin A, Szczylik C, Hutson TE, Michaelson MD, Gorbunova VA, Gore ME, Rusakov IG, et al: Comparative effectiveness of axitinib versus sorafenib in advanced renal cell carcinoma (AXIS): A randomised phase 3 trial. Lancet 378: 1931-1939, 2011.

5. Bennouna J, Sastre J, Arnold D, Österlund P, Greil R, Van Cutsem E, von Moos R, Viéitez JM, Bouché O, Borg C, et al; ML18147 Study Investigators: Continuation of bevacizumab after first progression in metastatic colorectal cancer (ML18147): A randomised phase 3 trial. Lancet Oncol 14: 29-37, 2013.

6. Rini BI, Melichar B, Ueda T, Grünwald V, Fishman MN, Arranz JA, Bair AH, Pithavala YK, Andrews GI, Pavlov D, et al: Axitinib with or without dose titration for first-line metastatic renal-cell carcinoma: A randomised double-blind phase 2 trial. Lancet Oncol 14: 1233-1242, 2013.

7. Grothey A, Van Cutsem E, Sobrero A, Siena S, Falcone A, Ychou M, Humblet Y, Bouché O, Mineur L, Barone C, et al; CORRECT Study Group: Regorafenib monotherapy for previously treated metastatic colorectal cancer (CORRECT): An international, multicentre, randomised, placebo-controlled, phase 3 trial. Lancet 381: 303-312, 2013.

8. Melichar B, Bracarda S, Matveev V, Alekseev B, Ivanov S, Zyryanov A, Janciauskiene R, Fernebro E, Mulders P, Osborne S, et al; BEVLiN Investigators: A multinational phase II trial of bevacizumab with low-dose interferon- $\alpha 2 \mathrm{a}$ as first-line treatment of metastatic renal cell carcinoma: BEVLiN. Ann Oncol 24: 2396-2402, 2013.

9. Fuchs CS, Tomasek J, Yong CJ, Dumitru F, Passalacqua R, Goswami C, Safran H, dos Santos LV, Aprile G, Ferry DR, et al; REGARD Trial Investigators: Ramucirumab monotherapy for previously treated advanced gastric or gastro-oesophageal junction adenocarcinoma (REGARD): An international, randomised, multicentre, placebo-controlled, phase 3 trial. Lancet 383: 31-39, 2014.

10. Van Cutsem E, Tabernero J, Lakomy R, Prenen H, Prausová J, Macarulla T, Ruff P, van Hazel GA, Moiseyenko V, Ferry D, et al: Addition of aflibercept to fluorouracil, leucovorin, and irinotecan improves survival in a phase III randomized trial in patients with metastatic colorectal cancer previously treated with an oxaliplatin-based regimen. J Clin Oncol 30: 3499-3506, 2012.

11. Melichar B and Nemcová I: Eye complications of cetuximab therapy. Eur J Cancer Care (Engl) 16: 439-443, 2007. 
12. Melichar B, Králíčková $P$, Hyšpler R, Kalábová $H$, Cerman J Jr, Holečková $\mathrm{P}$, Studentová $\mathrm{H}$ and Malírová E: Hypomagnesaemia in patients with metastatic colorectal carcinoma treated with cetuximab. Hepatogastroenterology 59: 366-371, 2012.

13. Melichar B, Hyšpler R, Tichá A, Kalábová H, Vitásková D, Zezulová M, Dvořák J, Cerman J Jr and Doležel M: Intestinal permeability in patients with metastatic colon cancer treated with patupilone. Clin Chem Lab Med 52: 1649-1655, 2014.

14. Melichar B, Dvorák J, Hyspler R and Zadák Z: Intestinal permeability in the assessment of intestinal toxicity of cytotoxic agents. Chemotherapy 51: 336-338, 2005.

15. Melichar B, Kohout P, Brátová M, Solichová D, Králícková $\mathrm{P}$ and Zadák Z: Intestinal permeability in patients with chemotherapy-induced stomatitis. J Cancer Res Clin Oncol 127: 314-318, 2001.

16. Geiger S, Lange V, Suhl P, Heinemann V and Stemmler HJ: Anticancer therapy induced cardiotoxicity: Review of the literature. Anticancer Drugs 21: 578-590, 2010.

17. Huddart RA, Norman A, Shahidi M, Horwich A, Coward D, Nicholls J and Dearnaley DP: Cardiovascular disease as a long-term complication of treatment for testicular cancer. J Clin Oncol 21: 1513-1523, 2003.

18. Meinardi MT, Gietema JA, van der Graaf WTA, van Veldhuisen DJ, Runne MA, Sluiter WJ, de Vries EG, Willemse PB, Mulder NH, van den Berg MP, et al: Cardiovascular morbidity in long-term survivors of metastatic testicular cancer. J Clin Oncol 18: 1725-1732, 2000.

19. Zagars GK, Ballo MT, Lee AK and Strom SS: Mortality after cure of testicular seminoma. J Clin Oncol 22: 640-647, 2004.

20. Kalábová H, Melichar B, Ungermann L, Doležal J, Krčmová L, Kašparová M, Plíšek J, Hyšpler R, Pecka M and Solichová D: Intima-media thickness, myocardial perfusion and laboratory risk factors of atherosclerosis in patients with breast cancer treated with anthracycline-based chemotherapy. Med Oncol 28: 1281-1287, 2011.
21. Melichar B, Kalábová H, Krcmová L, Kasparová M, Malírová E, Melicharová K, Pecka M, Hyspler R and Solichová D: Serum homocysteine, cholesterol, retinol, alpha-tocopherol, glycosylated hemoglobin and inflammatory response during therapy with bevacizumab, oxaliplatin, 5-fluorouracil and leucovorin. Anticancer Res 29: 4813-4820, 2009.

22. Šrámek V, Melichar B, Indrakova J, Študentová H, Kalábová H, Vrána D, Lukešová L, Adam T, Hlídková E, Juráňová J, et al: Risk factors of atherosclerosis in patients with history of breast cancer. Pteridines 24: 201-210, 2013.

23. Melichar B: Laboratory medicine and medical oncology: The tale of two Cinderellas. Clin Chem Lab Med 51: 99-112, 2013.

24. Floyd JD, Nguyen DT, Lobins RL, Bashir Q, Doll DC and Perry MC: Cardiotoxicity of cancer therapy. J Clin Oncol 23: 7685-7696, 2005

25. Melichar B, Gregor J, Solichová D, Lukes J, Tichý M and Pidrman V: Increased urinary neopterin in acute myocardial infarction. Clin Chem 40: 338-339, 1994.

26. Solichova D, Melichar B, Blaha V, Klejna M, Vavrova J, Palicka V and Zadak Z: Biochemical profile and survival in nonagenarians. Clin Biochem 34: 563-569, 2001.

27. Melichar B, Solichová D, Melicharová K, Malírová E, Cermanová $\mathrm{M}$ and Zadák Z: Urinary neopterin in patients with advanced colorectal carcinoma. Int J Biol Markers 21: 190-198, 2006.

28. Ewer MS, Vooletich MT, Durand JB, Woods ML, Davis JR, Valero V and Lenihan DJ: Reversibility of trastuzumab-related cardiotoxicity: New insights based on clinical course and response to medical treatment. J Clin Oncol 23: 7820-7826, 2005.

29. Schmidinger M, Zielinski CC, Vogl UM, Bojic A, Bojic M, Schukro C, Ruhsam M, Hejna M and Schmidinger H: Cardiac toxicity of sunitinib and sorafenib in patients with metastatic renal cell carcinoma. J Clin Oncol 26: 5204-5212, 2008.

30. Dorff TB, Goldkorn A and Quinn DI: Targeted therapy in renal cancer. Ther Adv Med Oncol 1: 183-205, 2009. 